\title{
LncRNAs are altered in lung squamous cell carcinoma and lung adenocarcinoma
}

\author{
Bing Liu ${ }^{1, *}$, Yifei Chen ${ }^{1, *}$, Jiong Yang ${ }^{1}$ \\ ${ }^{1}$ Department of Respiratory Medicine, Zhongnan Hospital of Wuhan University, Wuhan, China \\ *These authors contributed equally to this work
}

Correspondence to: Jiong Yang, email: jiong.yang.znh@gmail.com

Keywords: lung squamous cell carcinoma, lung adenocarcinoma, long non-coding RNA, overall survival, gene regulation

Received: August 08, $2016 \quad$ Accepted: November 14, $2016 \quad$ Published: November 26, 2016

Copyright: Liu et al. This is an open-access article distributed under the terms of the Creative Commons Attribution License (CC-BY), which permits unrestricted use, distribution, and reproduction in any medium, provided the original author and source are credited.

\section{ABSTRACT}

Long non-coding RNAs (IncRNAs) have been implicated in pathogenesis of various cancers, including lung squamous cell carcinoma (LUSC) and lung adenocarcinoma (LUAD). We used cBioPortal to analyze IncRNA alteration frequencies and their ability to predict overall survival (OS) using 504 LUSC and 522 LUAD samples from The Cancer Genome Atlas (TCGA) database. In LUSC, 624 IncRNAs had alteration rates $>1 \%$ and $64>10 \%$. In LUAD 625 IncRNAs had alteration rates $>1 \%$ and $36>$ $10 \%$. Among those, 620 IncRNAs had alteration frequencies $>1 \%$ in both LUSC and LUAD, while 22 were LUSC-specific and 23 were LUAD-specific. Twenty IncRNAs had alteration frequencies $>10 \%$ in both LUSC and LUAD, while 44 were LUSC-specific and 16 were LUAD specific. Genome ontology and pathway analyses produced similar results for LUSC and LUAD. Two InCRNAs (IGF2BP2-AS1 and DGCR5) correlated with better OS in LUSC, and three (MIR31HG, CDKN2A-AS1 and LINC01600) predicted poor OS in LUAD. Chip-seq and luciferase reporter assays identified potential IGF2BP2AS1, DGCR5 and LINC01600 promoters and enhancers. This study presented IncRNA landscapes and revealed differentially expressed, highly altered IncRNAs in LUSC and LUAD. LncRNAs that act as oncogenes and IncRNA-regulating transcription factors provide novel targets for anti-lung cancer therapeutics.

\section{INTRODUCTION}

Lung cancers cause the most cancer deaths worldwide, and 5 -year overall survival rates remain below $15 \%[1,2]$. Lung cancers include non-small cell (NSCLC) and small cell lung cancer (SCLC), with more than $80 \%$ of lung cancer patients suffering from NSCLC [1]. Lung squamous carcinoma (LUSC) and lung adenocarcinoma (LUAD) are the most common NSCLCs [3]. Non-coding RNAs, transcriptionally active genes that do not code for proteins, account for more than $80 \%$ of all genes. Long non-coding RNAs (lncRNAs) are non-coding RNAs longer than 200 nucleotides [4]. They regulate many normal and diseaserelated cellular functions, including cancer-related processes, and may predict overall survival in some type of cancers [5].

Many lncRNAs are biomarkers in lung cancer. Metastasis-associated lung adenocarcinoma transcript 1 (MALAT-1) may promote lung cancer metastasis to the brain via epithelial-mesenchymal transition, and was both highly expressed and associated with poor prognosis in
NSCLC [6]. PVT1 was upregulated in NSCLC tissues, was associated with tumor stage and local metastasis, and is a potential therapeutic target [7]. HOTAIR was highly expressed in advanced stage lung cancers. It might promote cancer cell migration and aggression, and was correlated with metastasis and poor prognosis $[8,9]$. ANRIL was also upregulated in NSCLC tumor tissues, and was positively associated with tumor stage, size and metastasis $[10,11]$. Although many studies have associated lncRNAs with NSCLC, most have involved small sample sizes, with little insight into lncRNA mechanisms of action and prognostic values. The Cancer Genome Atlas (TCGA) is a public database (http://cancergenome.nih.gov/) that includes 33 cancer types, along with related clinical and genomic data. The cBioPortal (http://cBioPortal.org) is a web-based public tool for exploring, visualizing, and analyzing TCGA and other cancer genetic data $[12,13]$. In this study, we used the TCGA database and cBioPortal tool to analyze lncRNA alterations and survival prediction values in both LUSC and LUAD. We also assessed the 
Gene ontologies $(G O)$ and important pathways associated with these selected lncRNAs and their coexpressed genes. Potential promoters and enhancers of the lncRNAs, IGF2BP2-AS1, DGCR5, and LINC01600 were identified and analyzed (Supplementary Figure S1).

\section{RESULTS}

\section{LncRNAs were highly altered in LUSC and LUAD}

To identify lncRNAs with high alteration frequencies in LUSC and LUAD, we downloaded the current lncRNA database from HUGO (www.HUGO.org), which includes 2772 lncRNAs (Supplementary Table S1). 2745 of these were recognized in cBioPortal (Supplementary Table S2), and their alternation frequencies were assessed in the LUSC and LUAD datasets. 624 and 64 lncRNAs had alteration rates $>1 \%$ and $>10 \%$, respectively, in LUSC (Supplementary Table S3, Table 1). 625 and 36 lncRNAs had alteration rates $>1 \%$ and $>10 \%$, respectively, in LUAD (Supplementary Table S4, Table 2). A venn diagram was used to determine commonly and differentially altered lncRNAs. In a venn diagram analysis, 620 of those lncRNAs with alteration frequencies $>1 \%$ were commonly altered in both LUSC and LUAD (Figure 1A), 22 lncRNAs were LUSC-specific and 23 were LUAD-specific. Of the lncRNAs with alteration frequencies $>10 \%, 20$ were commonly altered, 44 were LUSC-specific and 16 were LUAD-specific (Figure 1B-1C).

Genetic analysis showed that lncRNAs with alteration frequencies $>10 \%$ in LUSC accumulated within several cytobands. 22 lncRNAs were in one cytoband (chr 3: p26.3), and 10 were in a second cytoband (Ch8: p23.3) (Table 3 ). There was no such cytoband accumulation phenomenon for LUAD lncRNAs. The 9 lncRNAs in chromosome 1, and 5 lncRNAs in chromosome 8 were distributed in different cytobands (Table 4).

\section{GO and pathway analysis of IncRNAs with alteration frequencies $>10 \%$}

The predicted functions of lncRNAs with alteration frequencies $>10 \%$ were explored using computational bioinformatics (LncRNA2Function v70 (GENCODE v15) http://mlg.hit.edu.cn/lncrna2function/index.jsp). In GO analysis, top ten cell component analysis showed five overlapped components: acrosomal vesicle, pole plasm, $p$ granule, germ plasm, chromatoid body (Figure 2A-2B). Molecular function analysis showed only two enriched terms (retinoic acid receptor binding and lysozyme activity) were found in the LUAD IncRNA list. Retinoic acid receptor binding function was common for LUSC and LUAD (Figure 2C-2D). Even though there were cellular component and molecular function differences between LUSC and LUAD, the top ten biological processes were identical for both lung cancer types (Figure 2E-2F). Similarly, the top two pathways were the same between LUSC and LUAD: beta defensins and defensins (Figure 2G-2H).

\section{LncRNAs predict overall survival in LUSC and LUAD}

cBioPortal results showed that out of all highlyaltered lncRNAS, only IGF2BP2-AS1 and DGCR5 potentially predicted better overall survival in LUSC (Figure 3A-3B), and only CDKN2A-AS1, MIR31HG and LINC01600 potentially predicted poor overall survival in LUAD (Figure 3C-3E). Combinations of these lncRNAs were further analyzed. The combination of IGF2BP2AS1 and DGCR5 in LUSC and the trio combination (CDKN2A-AS1, MIR31HG and LINC01600) in LUAD could improve patient outcome prediction capability (Figure 3F-3G). In LUSC patients, lncRNA alterations mainly included upregulation and amplification (Figure 3H). In LUAD patients, CDKN2A-AS1 and MIR31HG were mainly deleted, while LINC01600 was upregulated (Figure 3I). We also investigated the prediction value of these five lncRNAs in different genders. We found that these identified lncRNAs play their roles in different genders. Generally, for the LUSC patients, IGF2BP2-AS1 play its role in male patients. For the LUAD patients, MIR31HG and LINC01600 play their roles in female patients, while CDKN2A-AS1 play its role in male patients (Supplementary Figure S2). For the five selected lncRNAs, we also performed disease free survival analysis, we found that only IGF2BP2-AS1 could still predict better DFS in LUSC, while other lncRNAs failed to predict DFS in NSCLC (Supplementary Figure S3).

\section{Analysis of selected IncRNA-coexpressed genes in LUSC}

LncRNAs regulate genes via multiple mechanisms $[14,15]$. cBioPortal analysis identified genes coexpressed with IGF2BP2-AS1, DGCR5, CDKN2A-AS1, MIR31HG and LINC01600 (Table 5). Genes coexpressed with IGF2BP2-AS1 and DGCR5 in LUSC, were analyzed in PANTHER (http://pantherdb.org/) (Figure 4). GO analysis showed that the top two enriched molecular functions for genes coexpressed with IGF2BP2-AS1 were binding and catalytic activity (Figure 4A). The most enriched biological processes were cellular process, metabolic process, developmental process, biological adhesion, and biological regulation (Figure 4B). The most enriched pathways were Alzheimer disease-presenilin pathway, angiogenesis, biotin biosynthesis, CCKR signaling map, cytoskeletal regulation by Rho GTPase, gonadotropin-releasing hormone receptor pathway, inflammation mediated by chemokine and cytokine signaling pathway, nicotine degradation, PDGF signaling pathway, VEGF signaling pathway, and Wnt signaling pathway (Figure 4B). The top two molecular 
Table 1: LncRNAs with alteration frequency higher than $10 \%$ in LUSC

\begin{tabular}{|c|c|c|c|c|}
\hline LncRNA & Alteration & Alteration reason & Cytoband & Position \\
\hline IGF2BP2-AS1 & 0.54 & Amp; high exp & $\mathrm{p} 26.3$ & Ch3:185,712,528-185,729,787 \\
\hline LINC00888 & 0.46 & Amp & $\mathrm{p} 26.3$ & Ch3:183,447,608-183,456,013 \\
\hline LINC00501 & 0.44 & Amp & $\mathrm{p} 26.3$ & Ch3:177,294,442-177,323,418 \\
\hline LINC00578 & 0.44 & Amp & $\mathrm{p} 26.3$ & Ch3:177,441,921-177,752,305 \\
\hline LINC00887 & 0.40 & Amp; high exp & $\mathrm{p} 26.3$ & Ch3:194,296,465-194,312,803 \\
\hline LINC00884 & 0.38 & Amp & $\mathrm{p} 26.3$ & Ch3:194,487,454-194,518,279 \\
\hline LINC00969 & 0.38 & Amp & $\mathrm{p} 26.3$ & Ch3:195,658,062-195,739,964 \\
\hline LINC00885 & 0.38 & Amp & p26.3 & Ch3:196,142,636-196,160,890 \\
\hline LINC01192 & 0.35 & Amp & $\mathrm{p} 26.3$ & Ch3:163,127,923-163,361,563 \\
\hline EXOC3-AS1 & 0.33 & Amp; high exp & p15.33 & Ch5:441,498-443,160 \\
\hline LINC00880 & 0.29 & Amp & $\mathrm{p} 26.3$ & Ch3:157,081,667-157,123,004 \\
\hline LINC00881 & 0.29 & Amp & $\mathrm{p} 26.3$ & Ch3:157,089,881-157,101,135 \\
\hline CDKN2A-AS1 & 0.29 & Del & $\mathrm{p} 24.3$ & Ch9:21,966,929-21,967,751 \\
\hline LINC00886 & 0.28 & Amp & $\mathrm{p} 26.3$ & Ch3:156,747,346-156,817,062 \\
\hline PVT1 & 0.23 & High exp; amp & $\mathrm{p} 23.3$ & Ch8:127,794,533-128,101,253 \\
\hline BPESC1 & 0.2 & Amp; high exp & $\mathrm{p} 26.3$ & Ch3:139,104,185-139,125,171 \\
\hline DUBR & 0.18 & Amp; high exp & $\mathrm{p} 26.3$ & Ch3:107,240,692-107,326,964 \\
\hline LINC00635 & 0.18 & Amp; high exp & $\mathrm{p} 26.3$ & Ch3:107,840,228-107,882,000 \\
\hline LINC01194 & 0.18 & Amp & p15.33 & Ch5:12,574,857-12,804,363 \\
\hline MIR31HG & 0.17 & Del; high exp & $\mathrm{p} 24.3$ & Ch9:21,455,642-21,559,669 \\
\hline TUG1 & 0.16 & Amp; del & p13 & Ch22:30,970,677-30,979,395 \\
\hline LINC01565 & 0.16 & Amp; high exp & $\mathrm{p} 26.3$ & Ch3:128,572,000-128,576,086 \\
\hline CASC8 & 0.16 & Amp; high exp & $\mathrm{p} 23.3$ & Ch8:127,289,817-127,482,139 \\
\hline LINC00603 & 0.15 & Amp & p15.33 & Ch5:40,052,291-40,053,324 \\
\hline TUSC7 & 0.14 & Amp; high exp & $\mathrm{p} 26.3$ & Ch3:116,709,235-116,723,581 \\
\hline LINC00964 & 0.14 & High exp; amp & $\mathrm{p} 23.3$ & Ch8:124,848,737-124,954,328 \\
\hline LINC00623 & 0.13 & High exp; amp & p36.33 & Ch1:120,913,275-121,009,291 \\
\hline SNHG20 & 0.13 & Amp; high exp & $\mathrm{p} 13.3$ & Ch17:77,086,716-77,094,990 \\
\hline SNHG11 & 0.13 & High exp & $\mathrm{p} 13$ & Ch20:38,446,578-38,450,921 \\
\hline LINC00882 & 0.13 & Amp; high exp & $\mathrm{p} 26.3$ & Ch3:106,836,811-107,240,641 \\
\hline LINC00879 & 0.13 & Amp & $\mathrm{p} 26.3$ & Ch3:94,938,172-95,152,509 \\
\hline HPYR1 & 0.13 & Amp; high exp & $\mathrm{p} 23.3$ & Ch8:132,560,498-132,561,479 \\
\hline LINC00626 & 0.12 & High exp; amp & p36.33 & Ch1:168,786,939-168,792,886 \\
\hline MIR205HG & 0.12 & High exp; amp & p36.33 & Ch1:209,428,820-209,432,838 \\
\hline FAM66C & 0.12 & High exp; amp & p13.33 & Ch12:8,180,209-8,216,151 \\
\hline DGCR5 & 0.12 & High exp; amp & p13 & Ch22:18,970,514-19,031,242 \\
\hline LINC00488 & 0.12 & Amp; high exp & $\mathrm{p} 26.3$ & Ch3:109,178,165-109,185,257 \\
\hline FAM167A-AS1 & 0.12 & Del; high exp & $\mathrm{p} 23.3$ & Ch8:11,368,402-11,438,658 \\
\hline FAM95B1 & 0.12 & high exp; amp & $\mathrm{p} 24.3$ & Ch9:40,321,299-40,329,221 \\
\hline GAS5 & 0.11 & high exp & p36.33 & Ch1:173,863,900-173,868,882 \\
\hline LINC00937 & 0.11 & High exp; amp & p13.33 & Ch12:8,295,986-8,396,803 \\
\hline SNHG10 & 0.11 & High exp; & p13 & Ch14:95,532,297-95,534,872 \\
\hline LINC00662 & 0.11 & High exp; amp & p13.3 & Ch19:27,684,580-27,793,940 \\
\hline
\end{tabular}




\begin{tabular}{lllll} 
SNHG17 & 0.11 & High exp; & p13 & Ch20:38,420,588-38,435,353 \\
CECR7 & 0.11 & High exp; & p13 & Ch22:17,036,570-17,060,825 \\
SNHG15 & 0.11 & High exp; & p22.3 & Ch7:44,983,023-44,986,961 \\
FAM74A3 & 0.11 & High exp; & p24.3 & Ch9:66,976,520-66,976,991 \\
LOH12CR2 & 0.1 & High exp; amp & p13.33 & Ch12:12,355,406-12,357,067 \\
LINC00923 & 0.1 & High exp; amp & p13 & Ch15:97,572,185-97,874,550 \\
LINC00470 & 0.1 & High exp; amp & p11.32 & Ch18:1,254,383-1,408,344 \\
LINC00667 & 0.1 & High exp; & p11.32 & Ch18:5,237,826-5,246,508 \\
LINC00493 & 0.1 & High exp; & p13 & Ch20:18,567,347-18,569,563 \\
LINC00634 & 0.1 & Amp & p13 & Ch22:41,952,165-41,958,933 \\
LINC00636 & 0.1 & Amp & p26.3 & Ch3:107,883,248-107,928,907 \\
LINC00901 & 0.1 & Amp & p26.3 & Ch3:116,921,431-116,932,238 \\
DANCR & 0.1 & High exp; amp & p16.3 & Ch4:52,712,404-52,720,351 \\
HCG18 & 0.1 & High & p25.3 & Ch6:30,287,397-30,327,150 \\
BAALC-AS2 & 0.1 & High amp & p23.3 & Ch8:103,132,963-103,141,475 \\
CCAT1 & 0.1 & Amp & p23.3 & Ch8:127,207,866-127,219,088 \\
LINC00051 & 0.1 & Amp; high exp & p23.3 & Ch8:142,198,356-142,209,003 \\
SNHG6 & 0.1 & High amp & p23.3 & Ch8:66,921,684-66,926,398 \\
FAM66E & 0.1 & Del; high exp & p23.3 & Ch8:7,955,014-8,008,755 \\
LINC00032 & 0.1 & Del; amp; high exp & p24.3 & Ch9:27,245,684-27,282,793 \\
LINC00910 & 0.1 & High & p13.3 & h17:43,369,845-43,389,199 \\
\hline
\end{tabular}

\section{A}

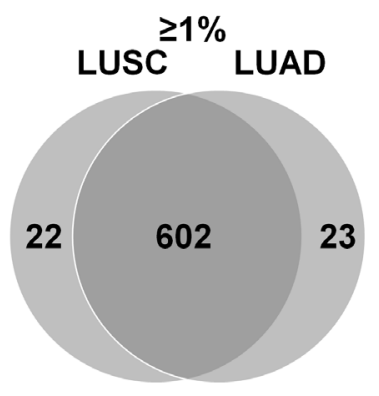

C

\begin{tabular}{lll}
\multicolumn{1}{c}{ LUSC } & \multicolumn{1}{c}{ Common } & \multicolumn{1}{c}{ LUAD } \\
BPESC1; CCAT1; CECR7; DANCR; & BAALC-AS2; CASC8; & ADAMTSL4-AS1; FALEC; \\
DGCR5; DUBR; FAM167A-AS1; & CDKN2A-AS1; EXOC3- & HAR1A; LINC00265; \\
FAM66C; FAM66E; FAM74A3; & AS1; GAS5; HCG18; & LINC00302; LINC00467; \\
FAM95B1; LINC00032; LINC00051; & HPYR1; IGF2BP2-AS1; & LINC00517; LINC00609; \\
LINC00470; LINC00488; LINC00493; & LINC00603; LINC00623; & LINC00624; LINC00894; \\
LINC00501; LINC00578; LINC00634; & LINC00626; LINC00662; & LINC00957; LINC01465; \\
LINC00635; LINC00636; LINC00667; LINC01194; MIR205HG; & LINC01600; PTCSC3; \\
LINC00879; LINC00880; LINC00881; & MIR31HG; PVT1; & TP53TG1; ZFAS1 \\
LINC00882; LINC00884; LINC00885; SNHG15; SNHG17; & \\
LINC00886; LINC00887; LINC00888; SNHG20; SNHG6 & \\
LINC00901; LINC00910; LINC00923; & & \\
LINC00937; LINC00964; LINC00969; & & \\
LINC01192; LINC01565; LOH12CR2; & & \\
SNHG10; SNHG11; TUG1; TUSC7 & &
\end{tabular}

B

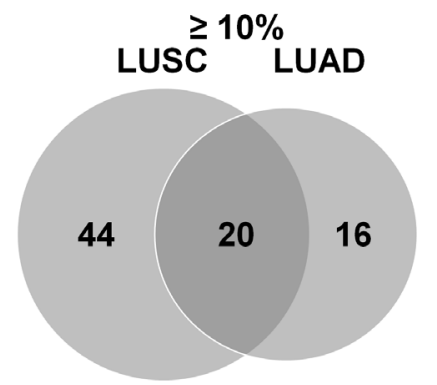


Table 2: LncRNAs with alteration frequency higher than $10 \%$ in LUAD

\begin{tabular}{|c|c|c|c|c|}
\hline LncRNA & Alteration & Alteration reason & Cytoband & Position \\
\hline EXOC3-AS1 & 0.29 & Amp; high exp & $\mathrm{p} 15.33$ & Chr5:441498-443160 \\
\hline PVT1 & 0.25 & Amp; high exp & q24.21 & Chr8:127794533-128101253 \\
\hline LINC00623 & 0.21 & Amp; high exp & $\mathrm{p} 11.2$ & Chr1:120912238-121009291 \\
\hline CDKN2A-AS1 & 0.2 & Del & $\mathrm{p} 21.3$ & chr9:21966929-21967754 \\
\hline LINC00467 & 0.18 & Amp; high exp & $\mathrm{q} 32.2$ & Chr1:211382755-211435333 \\
\hline HCG18 & 0.15 & High exp & $\mathrm{p} 22.1$ & Chr6:30287397-30327156 \\
\hline FALEC & 0.14 & Amp & $\mathrm{q} 21.3$ & Chr1:150515757-150518032 \\
\hline LINC01194 & 0.14 & Amp & $\mathrm{p} 15.2$ & Chr5:12574857-12805183 \\
\hline MIR31HG & 0.14 & Del & $\mathrm{p} 21.3$ & Chr9:21454268-21559698 \\
\hline LINC00624 & 0.13 & Amp & $\mathrm{q} 21.2$ & Chr1:147258885-147517875 \\
\hline ADAMTSL4-AS1 & 0.13 & Amp & $\mathrm{q} 21.3$ & Chr1:150560202-150574552 \\
\hline LINC00302 & 0.13 & Amp & $\mathrm{q} 21.3$ & Chr1:152655429-152656805 \\
\hline LINC00609 & 0.13 & Amp & $\mathrm{q} 13.2$ & Chr14:36070427-36165288 \\
\hline PTCSC3 & 0.13 & Amp & $\mathrm{q} 13.2$ & Chr14:36135710-36176651 \\
\hline LINC00662 & 0.13 & High exp & q11 & Chr19:27684580-27793940 \\
\hline LINC00957 & 0.13 & Amp; high exp & p13 & Chr7:44039049-44044296 \\
\hline LINC00626 & 0.12 & Amp; high exp & $\mathrm{q} 24.2$ & Chr1:168786939-168792886 \\
\hline GAS5 & 0.12 & Amp; high exp & $\mathrm{q} 25.1$ & Chr1:173863900-173868882 \\
\hline SNHG15 & 0.12 & Amp; high exp & $\mathrm{p} 13$ & Chr7:44983023-44986961 \\
\hline BAALC-AS2 & 0.12 & Amp; high exp & $\mathrm{q} 22.3$ & Chr8:103132963-103141475 \\
\hline LINC00517 & 0.11 & Amp & $\mathrm{q} 21.1$ & Chr14:37896060-37902372 \\
\hline SNHG20 & 0.11 & High exp & $\mathrm{q} 25.3$ & Chr17:77086716-77094990 \\
\hline HAR1A & 0.11 & Amp; high exp & $\mathrm{p} 13$ & Chr20:63102205-63104386 \\
\hline LINC00603 & 0.11 & Amp & p13.1 & Chr5:40052291-40053324 \\
\hline LINC00265 & 0.11 & Amp; high exp & $\mathrm{p} 14.1$ & Chr7:39733632-39793092 \\
\hline CASC8 & 0.11 & Amp & $\mathrm{q} 24.13$ & Chr8:127289817-127482139 \\
\hline HPYR1 & 0.11 & Amp; high exp & $\mathrm{q} 24.22$ & Chr8:132560498-132561479 \\
\hline LINC01465 & 0.1 & High exp & $\mathrm{q} 14.1$ & Chr12:62601751-62603690 \\
\hline SNHG17 & 0.1 & High exp & q12 & Chr20:38420588-38435353 \\
\hline ZFAS1 & 0.1 & High exp & $\mathrm{q} 13.13$ & Chr20:49278178-49295738 \\
\hline TP53TG1 & 0.1 & High exp & $\mathrm{q} 21.12$ & Chr7:87325225-87345515 \\
\hline SNHG6 & 0.1 & High exp & q13.1 & Chr8:66921684-66926398 \\
\hline LINC00894 & 0.1 & Amp; high exp & $\mathrm{q} 28$ & ChrX:149938628-150224580 \\
\hline IGF2BP2-AS1 & 0.1 & High exp & p26.3 & Chr3:185712528-185729787 \\
\hline LINC01600 & 0.1 & High exp & $\mathrm{p} 25.3$ & Chr6:2,621,913-2634603 \\
\hline MIR205HG & 0.1 & Amp; high exp & p36.33 & Chr1:209428820-209432838 \\
\hline
\end{tabular}

functions for genes coexpressed with DGCR5 were also binding and catalytic activity (Figure 4D). The top three enriched biological processes were cellular process, metabolic process and localization (Figure 4E). The most enriched biological processes were glycolysis and Parkinson's disease (Figure 4F).

\section{Analysis of selected IncRNA-coexpressed genes in LUAD}

Genes coexpressed with CDKN2A-AS1, MIR31HG and LINC01600 were analyzed. The most important molecular functions for both CDKN2A- 
AS1- and MIR31HG-coexpressed genes were binding and catalytic activity (Figure 5A and 5D). The top two enriched biological pathways were cellular process and metabolic process (Figure 5B and 5E), and the most enriched pathway was the P53 pathway (Figure 5C and 5F). GO and pathway analysis of LINC01600coexpressed genes showed that catalytic activity was the only enriched molecular function (Figure 5G). Cellular process, localization and metabolic process were the primary biological functions (Figure $5 \mathrm{H}$ ), and vasopressin synthesis was the only enriched pathway (Figure 5I).

CDKN2A-AS1 and MIR31HG are not far from one another on the same chromosome (Figure 5J), and three coexpressed genes, again on the same chromosome, were common to both: IFNE, CDKN2A and MTAP. cBioPortal analysis results showed that all three coexpressed genes shared very similar alteration patterns with CDKN2AAS1 and MIR31HG (Figure 5K). MTAP was the only gene located between CDKN2A-AS1 and MIR31HG. CDKN2A was next to, and partially overlapped with, CDKN2A-AS1, and IFNE was located in the MIR31HG intron (Figure 5K).

\section{LUSC}

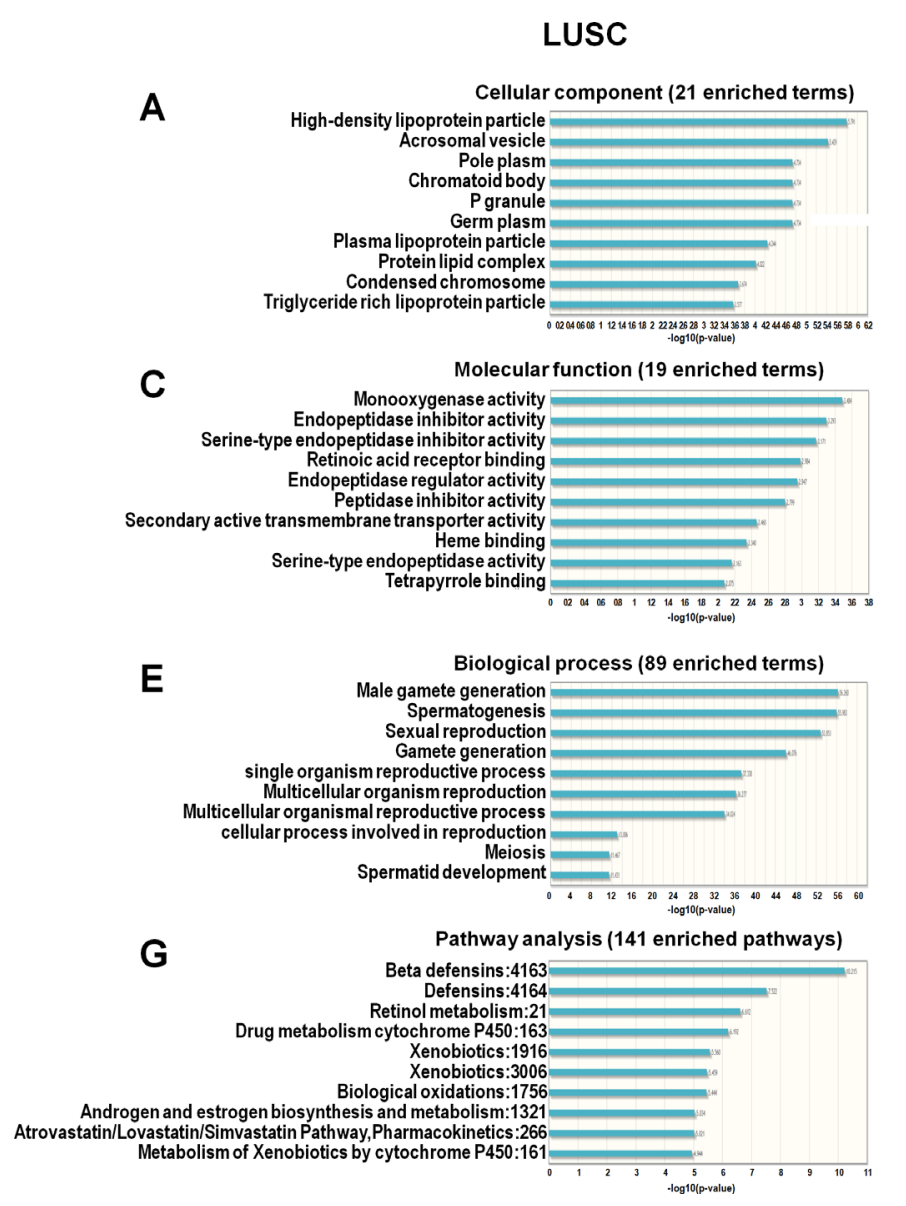

\section{Regulation of highly expressed IncRNAs}

LncRNAs are regulated through regulatory regions similar to protein-coding genes, and these regulatory regions can be predicted by chip-seq analysis. IGF2BP2AS1, DGCR5, and LINC01600 alterations were due to upregulation, so we evaluated how these IncRNAs were regulated using the ENCODE project database. There are many chip-seq datasets specific to the A549 cell line in the ENCODE database, including Pol II, H3K4Me2, H3K4Me3, and H3K27ac chip-seq data. Peaks in these data could help identify potential lncRNA promoters and enhancers [16]. CpG island hypomethylation is associated with gene overexpression [17], and conserved sequences also indicate regulatory regions [18]. We analyzed these predictors using IGV software. Promoters and potential enhancers of the three LUAD-associated IncRNAs were identified (Figure 6A-6C). Promoters were defined as $1 \mathrm{~kb}$ upstream of the first exon. Results identified one potential enhancer in IGF2BP-AS1 and DGCR5 (Figure 6A-6B), and two potential enhancers in LINC01600 (Figure 6C). $\mathrm{CpG}$ island analysis showed $\mathrm{CpG}$ islands in IGF2BP2-

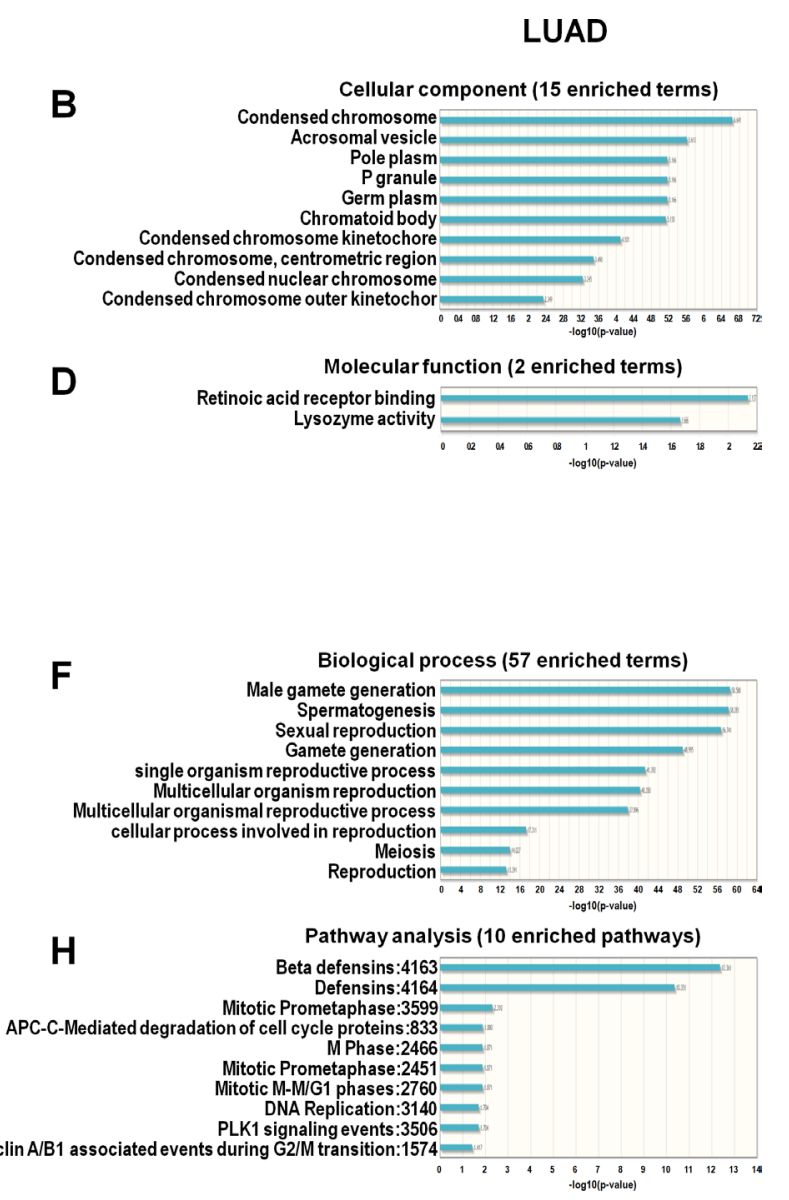

Figure 2: GO and pathway analysis of IncRNAs with alteration frequencies $>10 \%$ in LUSC and LUAD. Cellular components enriched in LUSC (A) and LUAD (B). Molecular functions enriched in LUSC (C) and LUAD (D). Biological processes enriched in LUSC (E) and LUAD (F) Pathways enriched in LUSC (G) and LUAD (H). 
Table 3: Character of IncRNAs in LUSC

\begin{tabular}{|c|c|c|}
\hline Chromosome & Cytoband & LncRNA \\
\hline Chr 1 & Chr1: p36.33 & LINC00623; LINC00626; GAS5; MIR205HG \\
\hline Chr 3 & Chr3: p26.3 & $\begin{array}{l}\text { LINC00882; DUBR; LINC00635; LINC00636; LINC00488; TUSC7; } \\
\text { LINC00901; LINC01565; BPESC1; LINC00886; LINC00880; } \\
\text { LINC00881; LINC01192; LINC00501; LINC00578; LINC00888; } \\
\text { IGF2BP2-AS1; LINC00887; LINC00884; LINC00969; LINC00885; } \\
\text { LINC00879 }\end{array}$ \\
\hline Chr 4 & Chr4: p16.3 & DANCR \\
\hline Chr 5 & Chr5: p15.33 & LINC01194; LINC00603; EXOC3-AS1 \\
\hline Chr 6 & Chr6: p25.3 & HCG18 \\
\hline Chr 7 & Chr7: p22.3 & SNHG15 \\
\hline Chr 8 & Chr8: p23.3 & $\begin{array}{l}\text { BAALC-AS2; FAM167A-AS1; LINC00964; CCAT1; CASC8; } \\
\text { PVT1; HPYR1; LINC00051; SNHG6; FAM66E }\end{array}$ \\
\hline Chr 9 & Chr9: p24.3 & MIR31HG; CDKN2A-AS1; LINC00032; FAM95B1; FAM74A3 \\
\hline Chr 12 & Chr12: p13.33 & LOH12CR2; FAM66C; LINC00937 \\
\hline Chr 14 & Chr14: p13 & SNHG10 \\
\hline Chr 15 & Chr15: p13 & LINC00923 \\
\hline Chr 17 & Chr17: p13.3 & SNHG20; LINC00910 \\
\hline Chr 18 & Chr18: p11.32 & LINC00470; LINC00667 \\
\hline Chr 19 & Chr19: p13.3 & LINC00662 \\
\hline Chr 20 & Chr20: p13 & LINC00493; SNHG17; SNHG11 \\
\hline Chr 22 & Chr22: p13 & CECR7; DGCR5; TUG1; LINC00634 \\
\hline
\end{tabular}

AS1 and DGCR5 promoter and enhancer regions (Figure $6 \mathrm{~A}-6 \mathrm{~B})$. Conserved regions are more likely to be regulated and functionally important. Conservation score analysis showed that DGCR5 promoter and putative enhancer regions were highly conserved (Figure 6B). Promoter and enhancer activities were determined via luciferase reporter assay. The combination of DGCR5 promoter and putative enhancer regions exhibited increased luciferase activity. The combination of promoter and enhancer 1, but not enhancer 2 in LINC01600 drove luciferase activity. However, the combination of IGF2BP2-AS1 promoter and putative enhancer induced no luciferase activity (Figure 6D).

\section{DISCUSSION}

LncRNAs reportedly play essential roles in lung cancer and other cancers, and may be useful biomarkers for identifying high- and low-risk patients, and predicting overall survival $[19,20]$. In this study, we comprehensively analyzed highly altered lncRNAs via cBioPortal with data sourced from the TCGA database. We identified 624 lncRNAs in LUSC and 625 in LUAD with alteration frequencies $>1 \%$, and 44 lncRNAs in LUSC and 36 in LUAD with alteration rates $>10 \%$. A Venn diagram analysis showed that $96 \%$ of $\operatorname{lncRNAs}$ with alteration rates $>1 \%$, and 20 total lncRNAs with alteration rates $>10 \%$ were shared between LUSC and
LUAD. GO and pathway analyses shed light on both shared and unique lncRNA functions in LUSC and LUAD. IGF2BP2-AS1 and DGCR5 were correlated with better prognosis in LUSC, while CDKN2A-AS1, MIR31HG and LINC01600 predicted poor prognosis in LUAD. GO and pathway analyses of genes coexpressed with these lncRNAs suggested possible mechanisms by which lncRNAs could impact prognosis. IGF2BP2-AS1, DGCR5 and LINC01600 were most frequently upregulated, and we showed that regulation occurred through promoters and enhancers, similar to protein-coding genes (Supplementary Figure S1).

LncRNAs with alteration frequencies $>10 \%$ in LUSC accumulated within several cytobands. There were 22 lncRNAs in ch3: p26.3 and 10 in ch8: p23.3. Genes in these two regions might play important roles in LUSC, but not LUAD. There were few studies to investigate the relationship between cytoband and NSCLC. We found that there was a deep deletion in these cytobands. So we hypothesize that some risk factors (such as smoking) might cause the chromosome damage and thus deep deletion. This hypothesis needed further investigation. We failed to find out specific references for this finding. We found that there was a deep deletion in these cytobands. So we hypothesize that some risk factors (such as smoking) might cause the chromosome damage and thus deep deletion. These cytobands might be key regions 
for LUSC. There was no such accumulation observed in LUAD. While the functions of some specific lncRNAs have been investigated [21-24], the overall function of highly altered lncRNAs in NSCLC was still unclear. In this study, we used LncRNA2Function, a web-based software, to perform GO and pathway analysis [25], which revealed many similarities between LUSC and LUAD. In cell component analysis, both LUSC and LUAD shared condensed chromosome, acrosomal vesicle, pole plasm, P granule, germ plasm, and chromatoid body. The distinct LUSC cellular components were high-density lipoprotein particle, lipoprotein particle, protein lipid complex, and triglyceride rich lipoprotein particle. The cellular components unique to LUAD were condensed chromosome kinetochore, condensed chromosome, centrometric region, condensed nuclear chromosome, and condensed chromosome outer kinetochor. There were only two enriched molecular function terms (retinoic acid receptor binding and lysozyme activity) in LUAD. Retinoic acid receptor beta is a tumor suppressor, and a
A

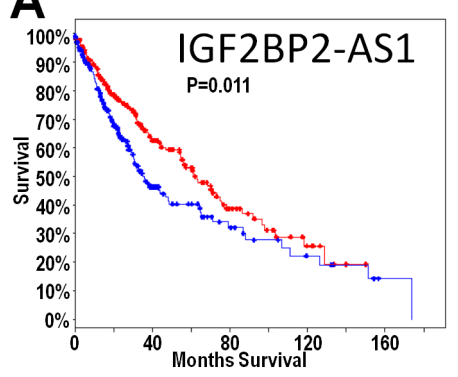

C

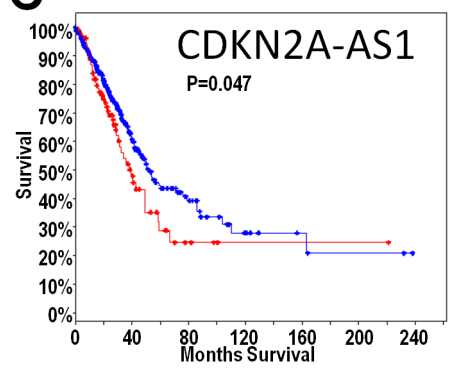

F

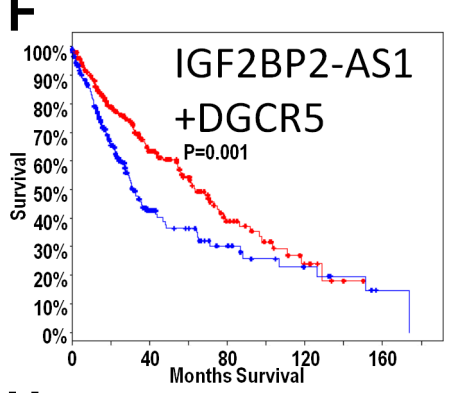

B
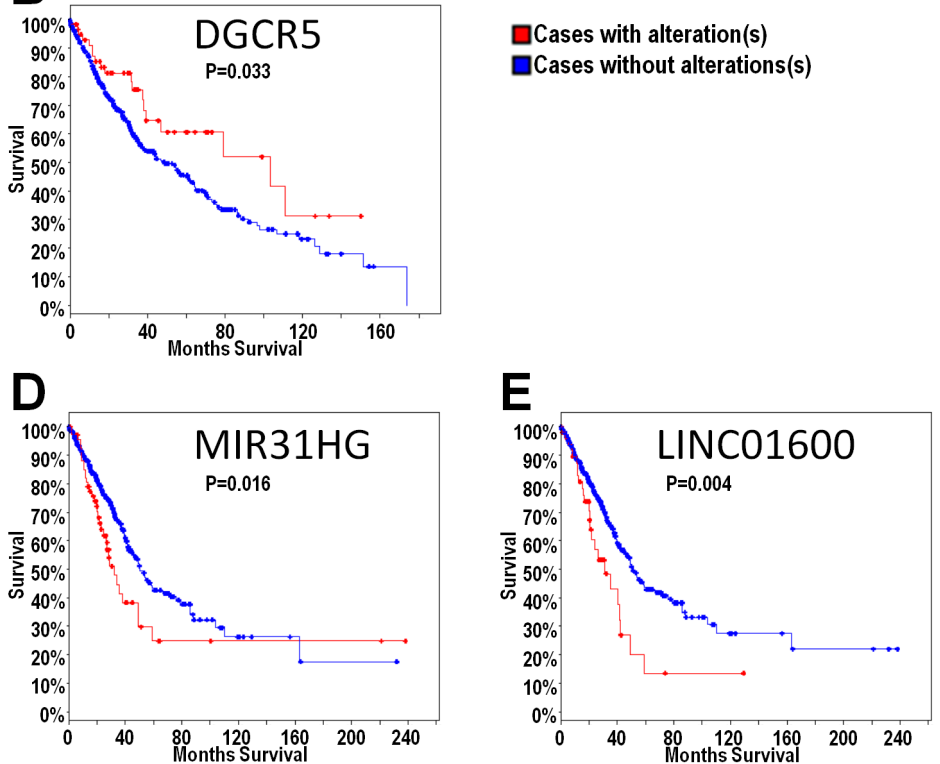

G

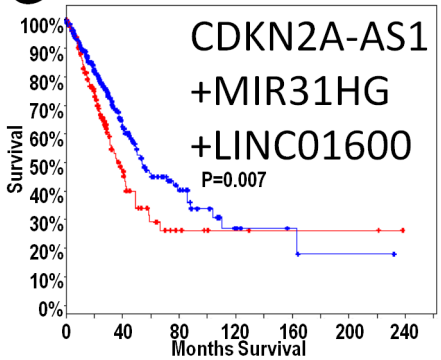

$\square$ Cases with alteration(s)
$\square$ Cases without alterations(s)

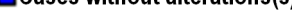

H

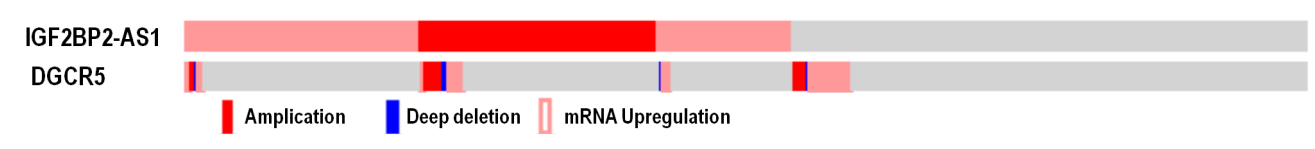

I

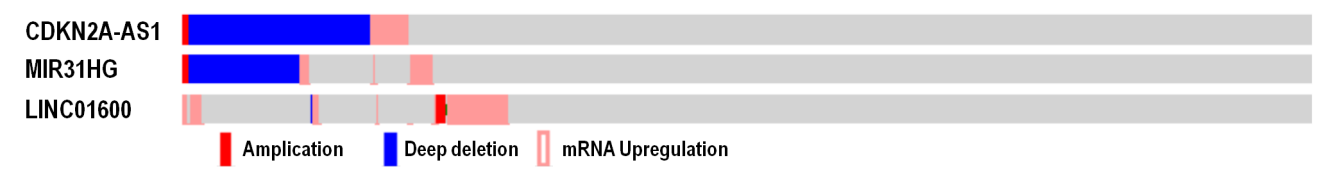

Figure 3: LncRNAs may predict overall survival in LUSC and LUAD. Overall survival analysis was presented as a KM curve by cBioPortal. Alteration of IGF2BP2-AS1 and DGCR5 alteration may predict good prognosis in LUSC (A-B) Alteration of CDKN2AAS1, MIR31HG and LINC01600 alterations may predict poor prognosis in LUAD (C-E) Combination of IGF2BP2-AS1 and DGCR5 alterations may predict good prognosis in LUSC (F). Combination of CDKN2A-AS1, MIR31HG and LINC01600 may predict poor prognosis in LUAD $(\mathbf{G})$. Upregulation and amplification were the primary IGF2BP2-AS1 and DGCR5 alterations (H). Deletion was the primary CDKN2A-AS1 and MIR31HG alteration, while LINC01600 was mainly upregulated (I). 


\begin{tabular}{|c|c|c|}
\hline Chromosome & Cytoband & LncRNA \\
\hline \multirow{7}{*}{ Chr 1} & Chr1: p11.2 & LINC00623 \\
\hline & Chr1: q21.2 & LINC00624 \\
\hline & Chr1: q21.3 & FALEC; ADAMTSL4-AS1; LINC00302 \\
\hline & Chr1: q24.2 & LINC00626 \\
\hline & Chr1: q25.1 & GAS5 \\
\hline & Chr1: p36.33 & MIR205HG \\
\hline & Chr1: q32.2 & LINC00467 \\
\hline \multirow[t]{2}{*}{ Chr 3} & Chr3: p26.3 & IGF2BP2-AS1 \\
\hline & Chr5: p15.2 & LINC01194 \\
\hline \multirow[t]{2}{*}{ Chr 5} & Chr5: p13.1 & LINC00603 \\
\hline & Chr5: p15.33 & EXOC3-AS1 \\
\hline \multirow{3}{*}{ Chr 6} & Chr6: p25.3 & LINC01600 \\
\hline & Chr6: p22.1 & HCG18 \\
\hline & Chr7: p14.1 & LINC00265 \\
\hline \multirow[t]{4}{*}{ Chr 7} & Chr7: p13; & LINC00957; SNHG15 \\
\hline & Chr7: q21.12 & TP53TG1 \\
\hline & Chr8: q22.3 & BAALC-AS2 \\
\hline & Chr8: q24.13 & CASC8 \\
\hline \multirow[t]{3}{*}{ Chr 8} & Chr8: q24.21 & PVT1 \\
\hline & Chr8: q24.22 & HPYR1 \\
\hline & Chr8: q13.1 & SNHG6 \\
\hline Chr 9 & Chr9: p21.3 & MIR31HG; CDKN2A-AS1; \\
\hline Chr12 & Chr12: q14.1 & LINC01465 \\
\hline \multirow{2}{*}{ Chr 14} & Chr14: q13.2 & LINC00609; PTCSC3 \\
\hline & Chr14: q21.1 & LINC00517 \\
\hline Chr 17 & Chr17: q25.3 & SNHG20 \\
\hline \multirow[t]{2}{*}{ Chr 19} & Chr19: q11 & LINC00662 \\
\hline & Chr20: q12 & SNHG17 \\
\hline \multirow[t]{2}{*}{ Chr 20} & Chr20: q13.13 & ZFAS1 \\
\hline & Chr20: p13 & HAR1A \\
\hline Chr X & ChrX: q28 & LINC00894 \\
\hline
\end{tabular}

prognostic indicator in stage I NSCLC [26]. Our results showed that lncRNAs in both LUSC and LUAD shared the retinoic acid receptor binding molecular function, which could be a target of these lncRNAs. The top nine enriched biological process terms were the same between LUSC and LUAD: male gamete generation, spermatogenesis, sexual reproduction, gamete generation, single organism reproductive process, multicellular organism reproduction, multicellular organismal reproductive process, cellular process involved in reproduction, and meiosis. All these terms, except reproduction, fell under the multicellular organism reproduction term. Female and male LUSC and LUAD patients experience differential pathogeneses and prognoses, and reproduction and sexual hormones can affect lung cancer outcome. A prospective study investigated the associations between reproductive factors, hormone use, and lung cancer risk, and found that both endogenous and extraneous estrogen may drive pathogenesis [27]. Our results suggest that lncRNAs might play roles in reproduction, thus modifying NSCLC pathogenesis and prognosis. Detailed overall survival analysis by gender could help determine the roles of IncRNAs in NSCLC. In our study, we found that for the LUSC patients, IGF2BP2-AS1 play its role in male patients. For the LUAD patients, MIR31HG and LINC01600 play their roles in female patients, while 
Table 5: Coexpression gene list of IGF2BP2-AS1, DGCR5, CDKN2A-AS1, MIR31HG, and LINC01600

\begin{tabular}{ll}
\hline \multicolumn{1}{c}{ LncRNA } & \multicolumn{1}{c}{ Coexpressed gene } \\
\hline CDKN2A-AS1 & IFNE; CDKN2A; CDKN2B; MTAP \\
& ACTN1; ANXA2; ANXA2P1; ANXA2P2; ARNTL2; BCAR3; CAMK2N1; CDC42EP2; CDCP1; \\
MIR31HG & CDKN2A; E2F7; EFHD2; FOSL1; FRMD6; FSCN1; GAPDH; GJB3; IFNA1; IFNA13; IFNE; \\
& LAMC2; LINC00704; MTAP; PKM; PLCD3; PLEK2; PLIN3; PML; SERPINB5; SLCO4A1; TGFBI; \\
& TMEM171; TPM4; TRPA1; VEGFC; ZYX \\
LINC01600 & SPC25; TMPO-AS1; TIMELESS \\
& TMEM246; CCDC136; SND1-IT1; DACT2; FAXC; C6ORF10; LOC730101; FBXL21; FBN2; \\
& ARHGEF28; KCNIP4-IT1; ETV5; IGF2BP2; SENP2; TMEM41A; CLCN2; FAM131A; YEATS2; \\
& DVL3; SOX2; TRA2B; B4GALT4; MAGEF1; TRAK1; UGT1A5; ARHGAP8; PRR5-ARHGAP8; \\
& CRYBB33; PAK7; C17ORF82; ETV4; BLMH; LOC646214; SPTLC2; SOX21; DGKA; NAB2; \\
& DTX3; GPRC5D; TSPAN18; KC6 \\
& SEPT5; DGCR9; RTN4R; DGCR10; EBF4; DIRAS1; ATP8B3; ENO2 \\
\hline
\end{tabular}

CDKN2A-AS1 play its role in male patients. A bunch of lncRNAs have been shown to participate in NSCLC.

In pathway analyses, LUSC and LUAD shared the top two pathways: beta defensins and defensins. Beta defensins were detected in lung epithelial cells and epithelial cell-derived lung cancer cells. Serum beta defensin level could be a lung cancer diagnostic marker [26]. LncRNAs might influence both LUSC and LUAD through beta defensins pathway. LncRNAs could also affect LUSC or LUAD through other distinct pathways. The other eight pathways in LUSC were retinol metabolism, drug metabolism cytochrome $\mathrm{P} 450$, xenobiotics, biological oxidations, androgen and estrogen biosynthesis and metabolism, Atrovastatin/Lovastatin/ Simvastatin pathway, pharmacokinetics, and metabolism of xenobiotics by cytochrome P450. Retinoid might be associated with LUSC, but this needs further confirmation [28]. Cytochrome P450 was associated with cancer

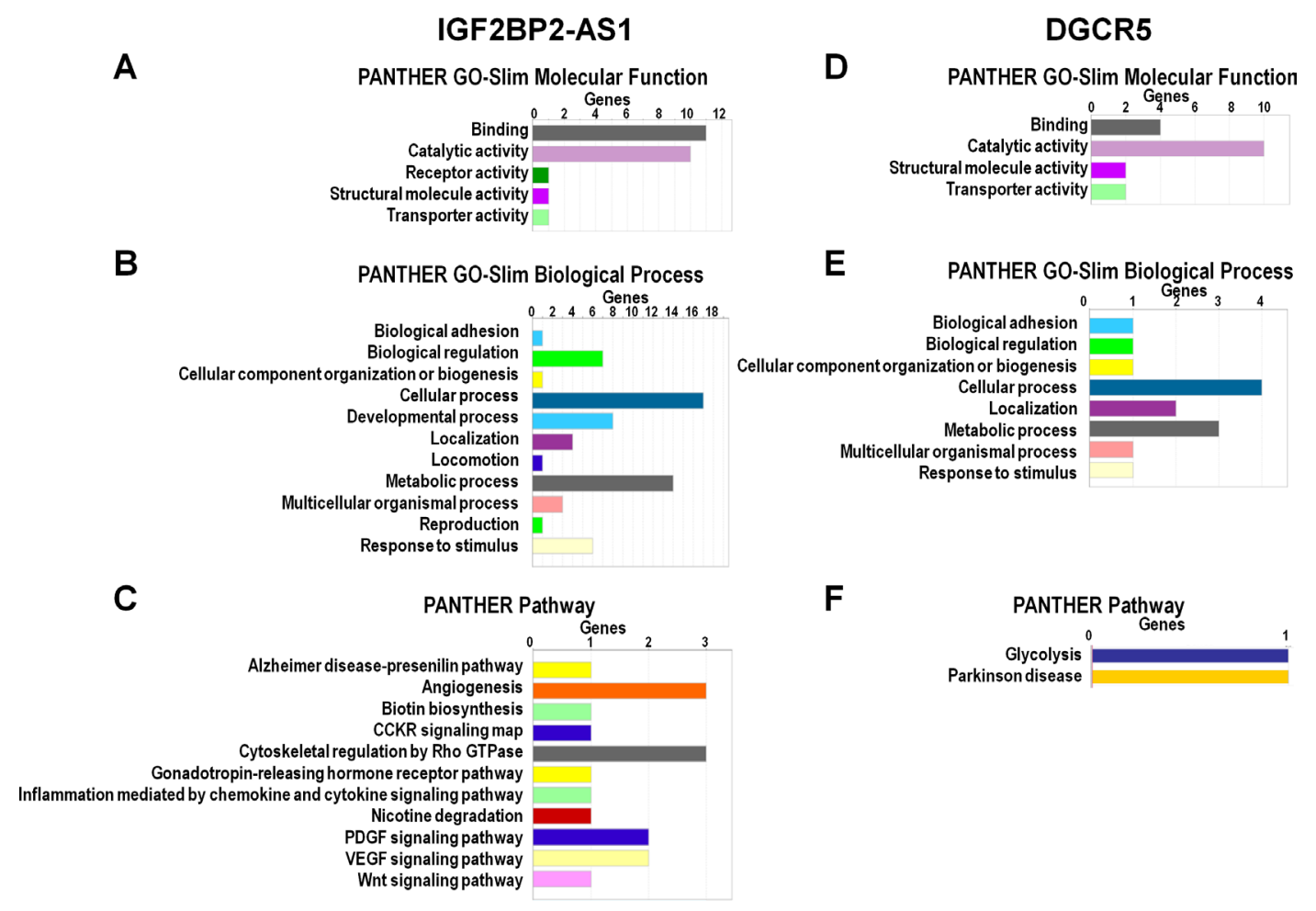

Figure 4: GO and pathway analysis of IGF2BP2-AS1- and DGCR5-coexpressed genes. GO and pathway analysis of IGF2BP2-AS1- (A-C) and DGCR5-coexpressed (D-F) protein-coding genes in LUSC according to molecular function, biological process, and pathways based on PANTHER analysis (http://pantherdb.org/). 
development, and polymorphisms are lung cancer risk factors [29]. Other pathways were also related to lung cancer development [30-34]. The remaining pathways in LUAD included mitotic prometaphase, APC-C-mediated degradation of cell cycle proteins, $\mathrm{M}$ phase, mitotic prometaphase, mitotic $\mathrm{M}-\mathrm{M} / \mathrm{G} 1$ phases, DNA replication, PLK1 signaling events, and cyclin A/B1 associated events during G2/M transition. All of these pathways have been associated with lung cancer [35-39].

In the present study, the overall survival was analyzed with respect to the NSCLC IncRNA landscape. IGF2BP2-AS1 and DGCR5 upregulation and amplification predicted better LUSC prognosis, possibly functioning as tumor suppressors. IGF2BP2AS1 is a lncRNA located in the IGF2BP2 antisense strand. IGF2BP2 promotes many cancer types [40-42], and IGF2BP2-AS1 might regulate IGF2BP expression, thus suppressing LUSC development and progression. DGCR5 is a DiGeorge syndrome-related gene [43], and its role in cancers is currently uncertain. MIR31HG, CDKN2AAS1 and LINC01600 predicted poor overall survival in LUAD. MIR31HG and CDKN2A-AS1 may act as tumor suppressors. MIR31HG promotes pancreatic ductal adenocarcinoma growth [44] and its downregulation was associated with bladder cancer development. In this study, we found that MIR31HG copy number deletion predicted poor prognosis in LUAD. CDKN2A-AS1 is an intronic lncRNA in the CDKN2A antisense strand, and is a tumor suppressor in NSCLC [45-47]. So it is very possible that CDKN2A-AS1 could regulate CDKN2A to affect lung cancer development. It seemed incompatible as for CDKN2A-AS1 and MIR31HG. We hypothesize that these two lncRNAs might help maintain the anti-cancer microenviroment or other similar mechanism. LINC01600 is an intergenic non-protein coding RNA, and very few studies have investigated its role in cancers.

LncRNAs can regulate proximal gene expression as well as that of genes farther away. We identified and investigated genes coexpressed with IGF2BP2-AS1, DGCR5, MIR31HG, CDKN2A-AS1 and LINC01600. GO-slim biological process analysis showed that both IGF2BP2-AS1 and DGCR5-coexpressed genes clustered within biological adhesion, biological regulation, cellular component organization or biogenesis, cellular process, localization, metabolic process, multicellular organismal process, and response to stimulus. IGF2BP2-AS1 and DGCR5 might play roles during LUSC development through similar biological processes. In pathway

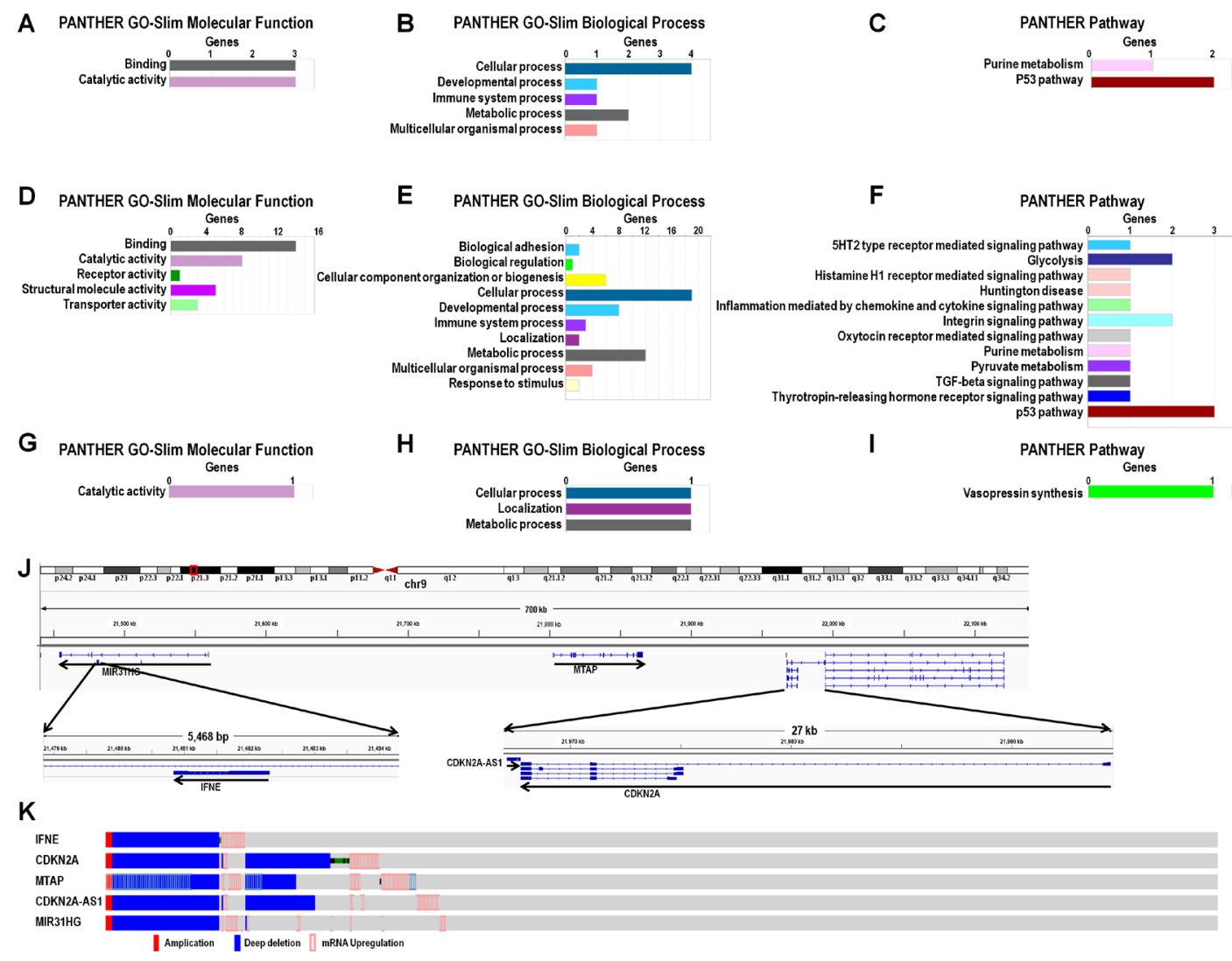

Figure 5: GO, pathway, and genomic analysis of CDKN2A-AS1, MIR31HG and LINC01600-coexpressed genes. Enrichment analysis of GO terms and pathways for CDKN2A-AS1- (A-C) MIR31HG- (D-F)., and LINC01600-coexpressed (G-I) protein-coding genes in LUAD based on PANTHER (http://pantherdb.org/). CDKN2A-AS1, MIR31HG and MTAP genomic positions (J) Main IFNE, CDKN2A, MTAP, MIR31HG and CDKN2A-AS1 alterations (K). 
analysis, we found that IGF2BP2-AS1-coexpressed genes participated in many pathways already associated with LUSC, such as angiogenesis [48], biotin biosynthesis [49], CCKR signaling map [50], cytoskeletal regulation by Rho GTPase [51], gonadotropin-releasing hormone receptor pathway [52], inflammation mediated by chemokine and cytokine signaling [53], nicotine degradation [53], PDGF signaling [54], VEGF signaling [55], and Wnt signaling [56]. There were only two enriched pathways associated with the DGCR5-coexpressed genes: glycolysis and Parkinson's disease. Glycolysis is already associated with NSCLC development and prognosis [57, 58].

$M I R 31 H G$ and $C D K N 2 A-A S 1$ are on the same chromosome. Three genes were located between these two
lncRNA genes: IFNE, MTAP, and CDKN2A, all of which are associated with cancer $[46,59,60]$. MIR31HG is a lncRNA that may be a tumor suppressor in glioblastoma [61]. MIR31HG downregulation promotes cell proliferation and was correlated with poor prognosis in gastric cancer [62]. In this study, we found that MIR31HG deletion predicted poor prognosis in LUAD.

LncRNA regulation mechanisms are still unclear, but they may be regulated similarly to protein-coding genes $[15,63]$. Previous studies showed that actively transcribed lncRNAs could be marked by RNA polymerase II (Pol II) and trimethylation of lysine 4 of histone $\mathrm{H} 3$ (H3K4me3) [64]. Dimethylation of histone $\mathrm{H} 3$ at lysine 4 (H3K4Me2) helped predict promoters and enhancers [65], and H3K27ac

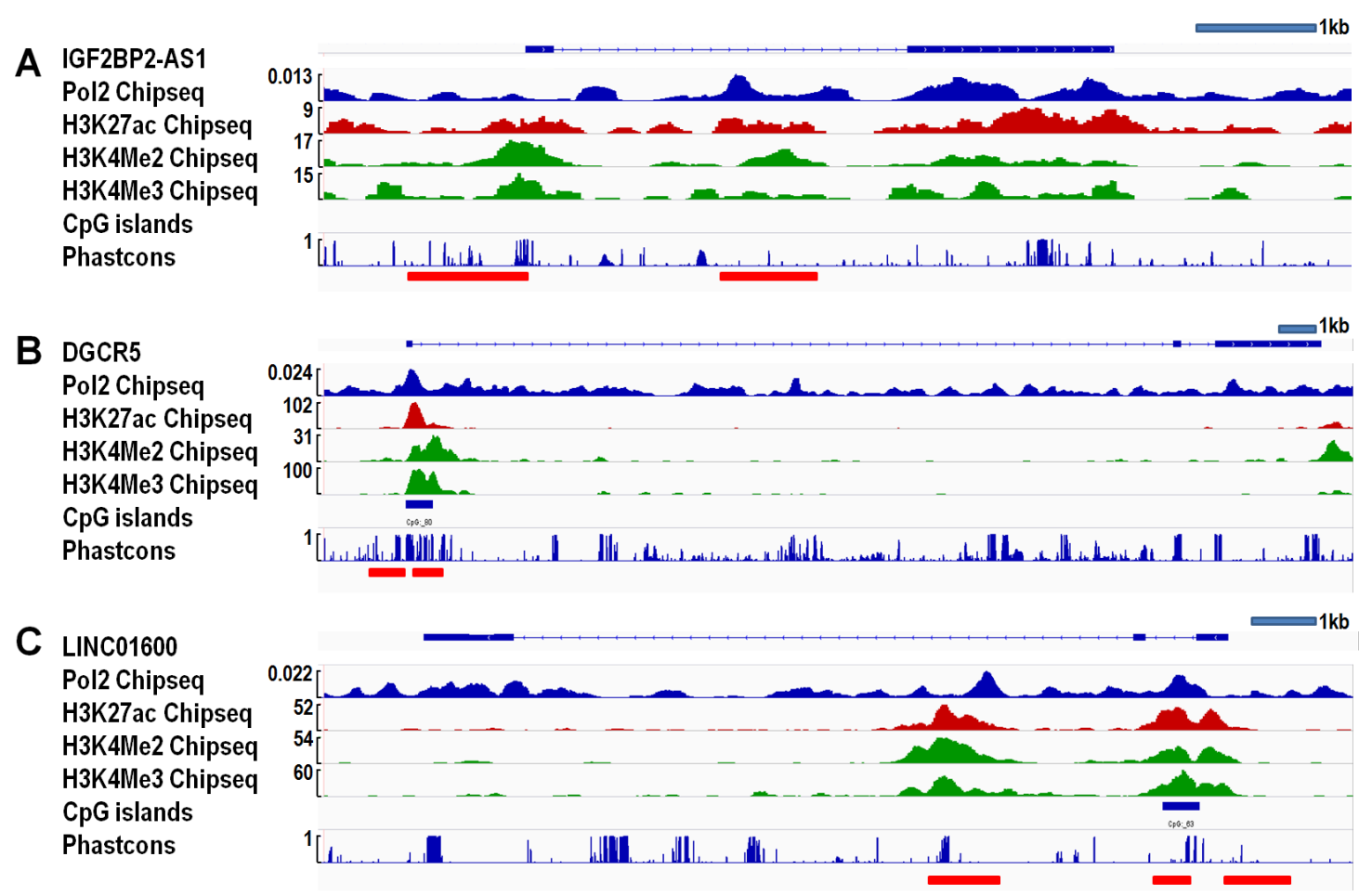

D

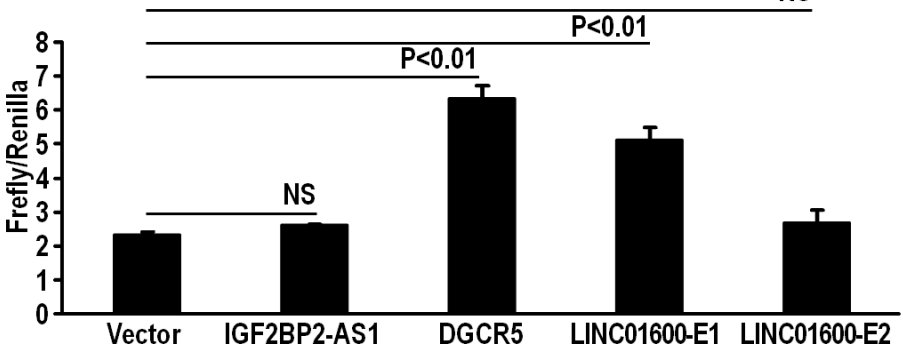

Figure 6: IGF2BP2-AS1, DGCR5, and LINC01600 promoter and enhancer analysis. Chip-seq, CpG island, and conservation score analysis of IGF2BP2-AS1 promoter and enhancer; one potential enhancer was found via Chip-seq peaks (A). Chip-seq, CpG island, and conservation score analysis of DGCR5 promoter and enhancer; one potential enhancer was found via Chip-seq peaks, CpG island and high conservation score (B). Chip-seq, CpG island, and conservation score analysis of LINC01600 promoter and enhancer; two potential enhancers were found, one via Chip-seq peaks, CpG island and high conservation score (E1), and the second via Chip-seq peaks alone (E2) (C). Relative luciferase activity of IGF2BP2-AS1, DGCR5, and LINC01600 promoter and enhancer (D). DGCR5 promoter and enhancer, and LINC01600 promoter and enhancer 1 showed increased relative luciferase activity (triplicates, mean \pm SEM). Data represented three independent experiments with similar results. 
helped identify active enhancers [66]. $\mathrm{CpG}$ island and conservation analysis could also help to identify regulatory regions $[17,18]$. Chip-seq data from the ENCODE project [16] were viewed in IGV software [67], integrating both $\mathrm{CpG}$ island data and conservation scores. In this study, we identified putative enhancers of IGF2BP2-AS1, DGCR5, and LINC01600. We confirmed that the DGCR5 and LINC01600 promoters and enhancers exhibited elevated activity in a luciferase reporter assay. This suggests that transcription factors could bind to these regulatory regions to direct their expression, thus affecting lung cancer development.

This study had several limitations. First, data were sourced from TCGA database and analyzed with cBioPortal. Even though there were more than 500 samples in both the LUAD and LUSC groups, our analyses may have missed potential biomarkers due to sample number restrictions. Similarly, we were unable to study some TCGA lncRNAs, because some were not recognized by cBioPortal. Additionally, because we chose the provisional database, database updates might affect our results and conclusions. Second, most patients in the TCGA database were white, and our study conclusions may have been different had we had access to Asian and African patient data. Third, lncRNA GO and pathway analyses were based on LncRNA2Function. LncRNA2Function is based on expression correlations between lncRNAs and protein-coding genes across 19 human normal tissues [25], and there might have been some false positive and negative function predictions. Fourth, lncRNA mechanisms of action in lung cancer development should be confirmed by knockout in lung cancer cells. Transgenic mice lacking these specific lncRNAs could also provide further functional information. Fifth, the transcription factors that regulate lncRNAs must still be identified.

In conclusion, this study presented a lncRNA landscape in NSCLC, and identified differentially expressed, highly altered lncRNAs in LUSC and LUAD. Two lncRNAs (IGF2BP2-AS1 and DGCR5) were correlated with better survival in LUSC and three (CDKN2A-AS1, MIR31HG, and LINC01600) could predict poor prognosis in LUAD. GO and pathway analysis of highly altered lncRNAs and their coexpressed genes helped identify potential mechanisms of action in lung cancer pathogenesis. Chip-seq and luciferase reporter analysis of the selected lncRNAs showed that they could be regulated through promoters and enhancers, similar to protein-coding genes. LncRNAs that act as oncogenes in NSCLC, and transcription factors that regulate cancerrelated lncRNAs provide potential novel targets for anticancer therapeutics.

\section{MATERIALS AND METHODS}

\section{Cell culture}

The lung cancer cell line, A549, was cultured in the Iscove's Modified Dulbecco's Medium (Corning,
Manassas, VA) with 10\% fetal bovine serum (FBS) (Fisher, Grand Island, NY), 100 units/mL penicillin and $100 \mu \mathrm{g} / \mathrm{mL}$ streptomycin. Cells were subcultured every two to three days. Confluence was maintained between $20 \%$ and $70 \%$.

\section{LncRNA analysis}

The lncRNA list was downloaded from the HUGO gene nomenclature committee (HGNC) (http://www. genenames.org/). 2745/2772 lncRNAs from the HUGO database were recognized by cBioPortal. There were 504 LUSC ("Lung Squamous Cell Carcinoma (TCGA, Provisional) 504 samples" dataset) and 522 LUAD ("Lung Adenocarcinoma (TCGA, Provisional) 522 samples" dataset) samples in the TCGA database (TCGA, Provisional). LncRNA somatic mutations, DNA copy number alterations (CNAs), and expression values were integrated into cBioPortal. LncRNA alteration frequency was defined as the number of cases with lncRNA alterations divided by the total number of cases. The primary goal was to identify the lncRNA alternation rate in these samples. LncRNAs with alternation rates $>10 \%$ were further analyzed to identify correlations with overall survival. Genes coexpressed with the selected lncRNAs were also identified by cBioPortal.

\section{Gene ontology (GO) and pathway analysis}

LncRNA2Function is an ontology-driven tool for exploring potential lncRNA functions to guide experimental and clinical investigations [25]. It is based on expression correlations between lncRNAs and proteincoding genes across 19 human normal tissues. LncRNAs with alteration frequencies $>10 \%$ were analyzed with this tool for GO and human biological pathways. The PANTHER (Protein ANalysis THrough Evolutionary Relationships) classification system was used to identify enriched GO terms and biological pathways for coexpressed genes.

\section{IGV-facilitated chromatin immunoprecipitation followed by sequencing (chip-seq), and LncRNA $\mathrm{CpG}$ island and conservation score analysis}

The Encyclopedia of DNA Elements (ENCODE) has constructed a comprehensive list of functional human genome elements, including those that act at the RNA level, and regulatory elements that control gene transcription (https://www.encodeproject.org/) [16]. We choose the GSE33213 series for the Pol II chipseq data and the GSE29611 series for the H3K4me2, H3K4me3 and H3K27ac chip-seq data in the A549 cell line. The Integrative Genomics Viewer (IGV) is a high performance visualization tool that has integrated the ENCODE database and human assemblies, such as $\mathrm{CpG}$ 
islands and various conservation tracks [67]. For the chipseq analysis, we loaded the ENCODE data into the IGV software. For the $\mathrm{CpG}$ island analysis, we chose "load from server" in the file option, then "annotations-sequence and regulation- $\mathrm{CpG}$ islands." For the conservation score analysis, we chose "load from server" in the file option, then "annotations-comparative genomics-Phastcons (vertebrate 46 way)."

\section{Vector construction and transfection, and luciferase reporter assay}

LncRNA IGF2BP2-AS1, DGCR5, and LINC01600 promoters and enhancers were cloned into the PGL3 vector. With specific primers (Supplementary Table S5) $20 \mu \mathrm{g}$ DNA from A549 cells was used as a PCR template. For IGF2BP2AS1 promoter and enhancer construction, we first PCRamplified the promoter region, then digested it with NheI and XhoI for $1 \mathrm{~h}$ at $37^{\circ} \mathrm{C}$. Digested DNA fragments were inserted into the similarly-digested PGL3 vector. Then we PCRamplified the enhancer region, and digested it with KpnI and MluI. Digested DNA fragments were cloned into the PGL3 vector containing the IGF2BP2-AS1 promoter. The DGCR5 promoter and enhancer were cloned into the PGL3 vector via a similar procedure using BgIII and HindIII for promoter construction, and MluI and XhoI enhancer construction. NheI and XhoI were used for LINC01600 promoter construction, and KpnI and MluI for enhancer construction. Plasmids were purified with Endo-Free Plasmid Maxi Kits (Omega Bio-tek). For transient transfection, $5 \times 10^{5}$ A549 cells/well were subcultured in 6-well plates for $18 \mathrm{~h}$. Medium containing penicillin/streptomycin in wells was replaced before transfection. Two $\mu \mathrm{g}$ PGL3-target firefly plasmid and $0.2 \mu \mathrm{g}$ PRL-SV40 renilla plasmid were added to $200 \mu \mathrm{l}$ cell culture medium (without FBS and penicillin/ streptomycin). $6.6 \mu \mathrm{g}$ polyethylenimine (PEI) was added into the solution and mixed gently by pipette for $10 \mathrm{~s}$. The DNAPEI-medium mixture was incubated at room temperature for $20 \mathrm{~min}$, then added to wells. The 6-well plate was swirled gently for $10 \mathrm{~s}$. Six h later, the solution was removed from wells and fresh medium was added. Twenty-four $\mathrm{h}$ after transfection, luminescence was measured using the DualLuciferase $^{\circledR}$ Reporter (DLR ${ }^{\mathrm{TM}}$ ) Assay System (Promega) following the manufacture's protocol.

\section{Statistical analysis}

All valid lncRNAs identified by cBioPortal were sorted by alteration frequency. LncRNAs with alternation frequency $>10 \%$ were selected for further analysis. To determine lncRNA alternation frequency differences between LUSC and LUAD, venn diagram analysis was performed (http:// bioinformatics.psb.ugent.be/webtools/Venn/). KaplanMeier analysis was performed with cBioPortal to determine overall survival. Student's $t$-test was used to determine statistical differences between two groups. $P<0.05$ was considered statistically significant.

\section{ACKNOWLEDGMENTS}

We thank the ENCODE Consortium, the Noam Shoresh lab at Broad Institute for generating H3K4me2, $\mathrm{H} 3 \mathrm{~K} 4 \mathrm{me} 3$ and H3K27ac chip-seq data in the A549 cell line, and the Terrence S. Furey lab at Duke University for generating pol II chip-seq data in the A549 cell line.

\section{CONFLICTS OF INTEREST}

The results here are in whole or part based upon data generated by the TCGA Research Network: http:// cancergenome.nih.gov/.

\section{REFERENCES}

1. Molina JR, Yang P, Cassivi SD, Schild SE, Adjei AA. Nonsmall cell lung cancer: epidemiology, risk factors, treatment, and survivorship. Mayo Clin Proc. 2008; 83:584-94.

2. Ryerson AB, Eheman CR, Altekruse SF, Ward JW, Jemal A, Sherman RL, Henley SJ, Holtzman D, Lake A, Noone AM, Anderson RN, Ma J, Ly KN, et al. Annual Report to the Nation on the Status of Cancer, 1975-2012, featuring the increasing incidence of liver cancer. Cancer. 2016; 122:1312-37.

3. Kawase A, Yoshida J, Ishii G, Nakao M, Aokage K, Hishida T, Nishimura M, Nagai K. Differences between squamous cell carcinoma and adenocarcinoma of the lung: are adenocarcinoma and squamous cell carcinoma prognostically equal? Jpn J Clin Oncol. 2012; 42:189-95.

4. Esteller M. Non-coding RNAs in human disease. Nat Rev Genet. 2011; 12:861-74.

5. Li J, Meng H, Bai Y, Wang K. Regulation of lncRNA and Its Role in Cancer Metastasis. Oncol Res. 2016; 23:205-17.

6. Shen L, Chen L, Wang Y, Jiang X, Xia H, Zhuang Z. Long noncoding RNA MALAT1 promotes brain metastasis by inducing epithelial-mesenchymal transition in lung cancer. J Neurooncol. 2015; 121:101-8.

7. Yang YR, Zang SZ, Zhong CL, Li YX, Zhao SS, Feng XJ. Increased expression of the lncRNA PVT1 promotes tumorigenesis in non-small cell lung cancer. Int J Clin Exp Pathol. 2014; 7:6929-35.

8. Nakagawa T, Endo H, Yokoyama M, Abe J, Tamai K, Tanaka N, Sato I, Takahashi S, Kondo T, Satoh K. Large noncoding RNA HOTAIR enhances aggressive biological behavior and is associated with short disease-free survival in human non-small cell lung cancer. Biochem Biophys Res Commun. 2013; 436:319-24.

9. Liu XH, Liu ZL, Sun M, Liu J, Wang ZX, De W. The long non-coding RNA HOTAIR indicates a poor prognosis and promotes metastasis in non-small cell lung cancer. BMC Cancer. 2013; 13:464. 
10. Lu Y, Zhou X, Xu L, Rong C, Shen C, Bian W. Long noncoding RNA ANRIL could be transactivated by c-Myc and promote tumor progression of non-small-cell lung cancer. Onco Targets Ther. 2016; 9:3077-84.

11. Lin L, Gu ZT, Chen WH, Cao KJ. Increased expression of the long non-coding RNA ANRIL promotes lung cancer cell metastasis and correlates with poor prognosis. Diagn Pathol. 2015; 10:14.

12. Cerami E, Gao J, Dogrusoz U, Gross BE, Sumer SO, Aksoy BA, Jacobsen A, Byrne CJ, Heuer ML, Larsson E, Antipin Y, Reva B, Goldberg AP, et al. The cBio cancer genomics portal: an open platform for exploring multidimensional cancer genomics data. Cancer Discov. 2012; 2:401-4.

13. Gao J, Aksoy BA, Dogrusoz U, Dresdner G, Gross B, Sumer SO, Sun Y, Jacobsen A, Sinha R, Larsson E, Cerami E, Sander C, Schultz N. Integrative analysis of complex cancer genomics and clinical profiles using the cBioPortal. Sci Signal. 2013; 6:pl1.

14. Cao J. The functional role of long non-coding RNAs and epigenetics. Biol Proced Online. 2014; 16:11.

15. Wu Z, Liu X, Liu L, Deng H, Zhang J, Xu Q, Cen B, Ji A. Regulation of lncRNA expression. Cell Mol Biol Lett. 2014; 19:561-75.

16. Consortium EP. An integrated encyclopedia of DNA elements in the human genome. Nature. 2012; 489:57-74.

17. Deaton AM, Bird A. CpG islands and the regulation of transcription. Genes Dev. 2011; 25:1010-22.

18. Villar D, Berthelot C, Aldridge S, Rayner TF, Lukk M, Pignatelli M, Park TJ, Deaville R, Erichsen JT, Jasinska AJ, Turner JM, Bertelsen MF, Murchison EP, et al. Enhancer evolution across 20 mammalian species. Cell. 2015; 160:554-66.

19. Huang J, Peng J, Guo L. Non-coding RNA: a new tool for the diagnosis, prognosis, and therapy of small cell lung cancer. J Thorac Oncol. 2015; 10:28-37.

20. Massuti B, Sanchez JM, Hernando-Trancho F, Karachaliou N, Rosell R. Are we ready to use biomarkers for staging, prognosis and treatment selection in early-stage non-small-cell lung cancer? Transl Lung Cancer Res. 2013; 2:208-21.

21. Loewen G, Jayawickramarajah J, Zhuo Y, Shan B. Functions of lncRNA HOTAIR in lung cancer. J Hematol Oncol. 2014; 7:90.

22. Li Z, Yu X, Shen J. ANRIL:a pivotal tumor suppressor long non-coding RNA in human cancers. Tumour Biol. 2016; 37:5657-61.

23. Li Z, Shen J, Chan MT, Wu WK. TUG1: a pivotal oncogenic long non-coding RNA of human cancers. Cell Prolif. 2016.

24. Chen J, Wang R, Zhang K, Chen LB. Long non-coding RNAs in non-small cell lung cancer as biomarkers and therapeutic targets. J Cell Mol Med. 2014; 18:2425-36.

25. Jiang Q, Ma R, Wang J, Wu X, Jin S, Peng J, Tan R, Zhang T, Li Y, Wang Y. LncRNA2Function: a comprehensive resource for functional investigation of human lncRNAs based on RNA-seq data. BMC Genomics. 2015; 16:S2.
26. Khuri FR, Lotan R, Kemp BL, Lippman SM, Wu H, Feng L, Lee JJ, Cooksley CS, Parr B, Chang E, Walsh GL, Lee JS, Hong WK, et al. Retinoic acid receptor-beta as a prognostic indicator in stage I non-small-cell lung cancer. J Clin Oncol. 2000; 18:2798-804.

27. Liu Y, Inoue M, Sobue T, Tsugane S. Reproductive factors, hormone use and the risk of lung cancer among middle-aged never-smoking Japanese women: a large-scale populationbased cohort study. Int J Cancer. 2005; 117:662-6.

28. Khuri FR, Lotan R. Retinoids in lung cancer: friend, foe, or fellow traveler? J Clin Oncol. 2004; 22:3435-7.

29. Vasile E, Tibaldi C, Leon GL, D'Incecco A, Giovannetti E. Cytochrome P450 1B1 (CYP1B1) polymorphisms are associated with clinical outcome of docetaxel in non-small cell lung cancer (NSCLC) patients. J Cancer Res Clin Oncol. 2015; 141:1189-94.

30. Liang YW, Chang CC, Hung CM, Chen TY, Huang TY, Hsu YC. Preclinical Activity of Simvastatin Induces Cell Cycle Arrest in G1 via Blockade of Cyclin D-Cdk4 Expression in Non-Small Cell Lung Cancer (NSCLC). Int J Mol Sci. 2013; 14:5806-16.

31. Siegfried JM. Smoking out reproductive hormone actions in lung cancer. Mol Cancer Res. 2014; 12:24-31.

32. Verma MK, Miki Y, Abe K, Nagasaki S, Niikawa H, Suzuki S, Kondo T, Sasano H. Co-expression of estrogen receptor beta and aromatase in Japanese lung cancer patients: gender-dependent clinical outcome. Life Sci. 2012; 91:800-8.

33. Christensen PM, Gotzsche PC, Brosen K. The sparteine/ debrisoquine (CYP2D6) oxidation polymorphism and the risk of lung cancer: a meta-analysis. Eur J Clin Pharmacol. 1997; 51:389-93.

34. Rusinova GG, Azizova TV, Viazovskaia NS, Gur'ianov M, Osovets IV, Glazkova. [Role of ancestral genotypes of the genes of xenobiotics biotransformation in susceptibility to lung cancer among Mayak workers]. Radiats Biol Radioecol. 2013; 53:344-54.

35. Yoon HE, Kim SA, Choi HS, Ahn MY, Yoon JH, Ahn SG. Inhibition of Plk1 and Pin1 by 5'-nitro-indirubinoxime suppresses human lung cancer cells. Cancer Lett. 2012; 316:97-104.

36. Xu C, Li S, Chen T, Hu H, Ding C, Xu Z, Chen J, Liu Z, Lei Z, Zhang HT, Li C, Zhao J. miR-296-5p suppresses cell viability by directly targeting PLK1 in non-small cell lung cancer. Oncol Rep. 2016; 35:497-503.

37. Allera-Moreau C, Rouquette I, Lepage B, Oumouhou N, Walschaerts M, Leconte E, Schilling V, Gordien K, Brouchet L, Delisle MB, Mazieres J, Hoffmann JS, Pasero $\mathrm{P}$, et al. DNA replication stress response involving PLK1, CDC6, POLQ, RAD51 and CLASPIN upregulation prognoses the outcome of early/mid-stage non-small cell lung cancer patients. Oncogenesis. 2012; 1:e30.

38. Mariatos G, Bothos J, Zacharatos P, Summers MK, Scolnick DM, Kittas C, Halazonetis TD, Gorgoulis VG. 
Inactivating mutations targeting the chfr mitotic checkpoint gene in human lung cancer. Cancer Res. 2003; 63:7185-9.

39. Erez A, Perelman M, Hewitt SM, Cojacaru G, Goldberg I, Shahar I, Yaron P, Muler I, Campaner S, Amariglio N, Rechavi G, Kirsch IR, Krupsky M, et al. Sil overexpression in lung cancer characterizes tumors with increased mitotic activity. Oncogene. 2004; 23:5371-7.

40. Ye S, Song W, Xu X, Zhao X, Yang L. IGF2BP2 promotes colorectal cancer cell proliferation and survival through interfering with RAF-1 degradation by miR-195. FEBS Lett. 2016; 590:1641-50.

41. Liu X, Chen Z, Zhao X, Huang M, Wang C, Peng W, Yin J, Li J, He G, Li X, Zhu X. Effects of IGF2BP2, KCNQ1 and GCKR polymorphisms on clinical outcome in metastatic gastric cancer treated with EOF regimen. Pharmacogenomics. 2015; 16:959-70.

42. Barghash A, Golob-Schwarzl N, Helms V, Haybaeck J, Kessler SM. Elevated expression of the IGF2 mRNA binding protein 2 (IGF2BP2/IMP2) is linked to short survival and metastasis in esophageal adenocarcinoma. Oncotarget. 2016; 7:49743-49750. doi: 10.18632/oncotarget.10439.

43. Sutherland HF, Wadey R, McKie JM, Taylor C, Atif U, Johnstone KA, Halford S, Kim UJ, Goodship J, Baldini A, Scambler PJ. Identification of a novel transcript disrupted by a balanced translocation associated with DiGeorge syndrome. Am J Hum Genet. 1996; 59:23-31.

44. Yang H, Liu P, Zhang J, Peng X, Lu Z, Yu S, Meng Y, Tong WM, Chen J. Long noncoding RNA MIR31HG exhibits oncogenic property in pancreatic ductal adenocarcinoma and is negatively regulated by miR-193b. Oncogene. 2016; 35:3647-57.

45. Zhang W, Zhu J, Bai J, Jiang H, Liu F, Liu A, Liu P, Ji G, Guan R, Sun D, Ji W, Yu Y, Jin Y, et al. Comparison of the inhibitory effects of three transcriptional variants of CDKN2A in human lung cancer cell line A549. J Exp Clin Cancer Res. 2010; 29:74.

46. Tam KW, Zhang W, Soh J, Stastny V, Chen M, Sun H, Thu K, Rios JJ, Yang C, Marconett CN, Selamat SA, LairdOffringa IA, Taguchi A, et al. CDKN2A/p16 inactivation mechanisms and their relationship to smoke exposure and molecular features in non-small-cell lung cancer. J Thorac Oncol. 2013; 8:1378-88.

47. Kim N, Song M, Kim S, Seo Y, Kim Y, Yoon S. Differential regulation and synthetic lethality of exclusive RB1 and CDKN2A mutations in lung cancer. Int J Oncol. 2016; 48:367-75.

48. Onn A, Bar J, Herbst RS. Angiogenesis inhibition and lungcancer therapy. Lancet Oncol. 2014; 15:124-5.

49. Scheerger SB, Zempleni J. Expression of oncogenes depends on biotin in human small cell lung cancer cells NCI-H69. Int J Vitam Nutr Res. 2003; 73:461-7.

50. Moody TW, Nuche-Berenguer B, Moreno P, Jensen RT. CI-988 Inhibits EGFR Transactivation and Proliferation
Caused by Addition of CCK/Gastrin to Lung Cancer Cells. J Mol Neurosci. 2015; 56:663-72.

51. Kim TY, Jackson S, Xiong Y, Whitsett TG, Lobello JR, Weiss GJ, Tran NL, Bang YJ, Der CJ. CRL4A-FBXW5mediated degradation of DLC1 Rho GTPase-activating protein tumor suppressor promotes non-small cell lung cancer cell growth. Proc Natl Acad Sci U S A. 2013; 110:16868-73.

52. Godbert B, Tiotiu A, Masias C, Martinet Y. [Inadequate secretion of beta-human chorionic gonadotropin in lung cancer]. Rev Pneumol Clin. 2013; 69:298-301.

53. Rivas-Fuentes S, Salgado-Aguayo A, Pertuz Belloso S, Gorocica Rosete P, Alvarado-Vasquez N, Aquino-Jarquin G. Role of Chemokines in Non-Small Cell Lung Cancer: Angiogenesis and Inflammation. J Cancer. 2015; 6:938-52.

54. Zhang C, Ding XP, Zhao QN, Yang XJ, An SM, Wang H, $\mathrm{Xu} \mathrm{L}$, Zhu L, Chen HZ. Role of a7-nicotinic acetylcholine receptor in nicotine-induced invasion and epithelial-tomesenchymal transition in human non-small cell lung cancer cells. Oncotarget. 2016; 7: 59199-59208. doi: 10.18632/oncotarget.10498.

55. Donnem T, Al-Shibli K, Al-Saad S, Busund LT, Bremnes RM. Prognostic impact of fibroblast growth factor 2 in non-small cell lung cancer: coexpression with VEGFR-3 and PDGF-B predicts poor survival. J Thorac Oncol. 2009; 4:578-85.

56. Fontanini G, Vignati S, Lucchi M, Mussi A, Calcinai A, Boldrini L, Chine S, Silvestri V, Angeletti CA, Basolo F, Bevilacqua G. Neoangiogenesis and p53 protein in lung cancer: their prognostic role and their relation with vascular endothelial growth factor (VEGF) expression. Br J Cancer. 1997; 75:1295-301.

57. Yang J, Li J, Le Y, Zhou C, Zhang S, Gong Z. PFKL/ miR-128 axis regulates glycolysis by inhibiting AKT phosphorylation and predicts poor survival in lung cancer. Am J Cancer Res. 2016; 6:473-85.

58. Chen HH, Chiu NT, Su WC, Guo HR, Lee BF. Prognostic value of whole-body total lesion glycolysis at pretreatment FDG PET/CT in non-small cell lung cancer. Radiology. 2012; 264:559-66.

59. Kryukov GV, Wilson FH, Ruth JR, Paulk J, Tsherniak A, Marlow SE, Vazquez F, Weir BA, Fitzgerald ME, Tanaka M, Bielski CM, Scott JM, Dennis C, et al. MTAP deletion confers enhanced dependency on the PRMT5 arginine methyltransferase in cancer cells. Science. 2016; 351:1214-8.

60. Matsumiya T, Prescott SM, Stafforini DM. IFN-epsilon mediates TNF-alpha-induced STAT1 phosphorylation and induction of retinoic acid-inducible gene-I in human cervical cancer cells. J Immunol. 2007; 179:4542-9.

61. Rajbhandari R, McFarland BC, Patel A, Gerigk M, Gray GK, Fehling SC, Bredel M, Berbari NF, Kim H, Marks MP, Meares GP, Sinha T, Chuang J, et al. Loss of tumor suppressive microRNA-31 enhances TRADD/ NF-kappaB signaling in glioblastoma. Oncotarget. 2015; 6:17805-16. doi: 10.18632/oncotarget.4596. 
62. Nie FQ, Ma S, Xie M, Liu YW, De W, Liu XH. Decreased long noncoding RNA MIR31HG is correlated with poor prognosis and contributes to cell proliferation in gastric cancer. Tumour Biol. 2016; 37:7693-701.

63. Wu SC, Kallin EM, Zhang Y. Role of H3K27 methylation in the regulation of IncRNA expression. Cell Res. 2010; 20:1109-16.

64. Guttman M, Amit I, Garber M, French C, Lin MF, Feldser D, Huarte M, Zuk O, Carey BW, Cassady JP, Cabili MN, Jaenisch R, Mikkelsen TS, et al. Chromatin signature reveals over a thousand highly conserved large non-coding RNAs in mammals. Nature. 2009; 458:223-7.
65. Wang $\mathrm{Y}, \mathrm{Li} \mathrm{X}, \mathrm{Hu} \mathrm{H} . \mathrm{H} 3 \mathrm{~K} 4 \mathrm{me} 2$ reliably defines transcription factor binding regions in different cells. Genomics. 2014; 103:222-8.

66. Creyghton MP, Cheng AW, Welstead GG, Kooistra T, Carey BW, Steine EJ, Hanna J, Lodato MA, Frampton GM, Sharp PA, Boyer LA, Young RA, Jaenisch R. Histone H3K27ac separates active from poised enhancers and predicts developmental state. Proc Natl Acad Sci U S A. 2010; 107:21931-6.

67. Robinson JT, Thorvaldsdottir H, Winckler W, Guttman M, Lander ES, Getz G, Mesirov JP. Integrative genomics viewer. Nat Biotechnol. 2011; 29:24-6. 